\title{
Patterns of Volatile Organic Compounds in Excrements of Preterm Neonates Detected by Ion Mobility Spectrometry Coupled to Multi-Capillary Columns
}

\section{Michelle Bous}

Saarland University Medical School

\section{Erol Tutdibi}

Saarland University Medical School

Nasenien Nourkami-Tutdibi

Saarland University Medical School

Elisabeth Kaiser

Saarland University Medical School

\section{Regine Stutz}

Saarland University Medical School

\section{Sascha Meyer}

Saarland University Medical School

Jörg Ingo Baumbach

Technical University Dortmund

\section{Michael Zemlin}

Saarland University Medical School

Sybelle Goedicke-Fritz ( $\sim$ Sybelle.goedicke-fritz@uks.eu )

Saarland University Medical School

\section{Research Article}

Keywords: Volatile organic compounds, ion mobility spectrometry, premature infant, biomarkers, 5methyl-2,1-methylethylcyclohexanol, 2-hexanone, 1-butanal, 2-pentylfuran, benzonitrile, detection, pediatrics, non-invasive diagnostics, VOC, IMS, neonatology, analytical science

Posted Date: February 24th, 2022

DOI: https://doi.org/10.21203/rs.3.rs-1361210/v1

License: (c) (i) This work is licensed under a Creative Commons Attribution 4.0 International License. Read Full License 
Page $2 / 22$ 


\section{Abstract \\ Background}

As neonates are susceptible for many diseases, establishing non-invasive diagnostic methods is desirable. We hypothesized that volatile organic compounds (VOCs) could be successfully measured in diaper samples.

\section{Methods}

We performed a feasibility study to investigate whether ambient air-independent headspace measurements of the VOC profiles of diapers from premature infants can be conducted using ion mobility spectrometer coupled with multi-capillary columns (B \& S Analytik GmbH, Dortmund, Germany).

\section{Results}

We analyzed 39 diapers filled with stool $(n=10)$ or urine $(n=20)$ respectively, using empty diapers as a control $(n=9)$. A total of 158 different VOCs were identified, and we classified the content of the diapers (urine or stool) according to their VOC profiles with a significance level of $p<0.05$.

\section{Discussion}

We have developed a novel method to study headspace VOC profiles of biosamples using ion mobility spectrometry coupled with multi-capillary columns. Using this method, we have characterized the VOC profiles of stool and urine of preterm neonates. Future studies are warranted to characterize specific VOC profiles in infections and other diseases of the preterm neonate, thus establishing quick and non-invasive diagnostics in the routine care of the highly vulnerable preterm and term neonates.

\section{Introduction}

Every tenth child worldwide is born prematurely ${ }^{1}$. A state of immaturity of all organ systems including the immune system is associated with prematurity demanding intensive medical care treatment. Diagnosis of infections are difficult in preterm infants as they are often linked to nonspecific symptoms. Diagnostic procedures are not merely painful for premature infants; but other major problems include the time to diagnosis and treatment, costs and sensitivity of existing diagnostic tests. In daily clinical practice, markers of systemic inflammation and immune responses like C-reactive protein (CrP), procalcitonin (PCT), Interleukin-6 (IL-6), and Interleukin-8 (IL-8), provide limited sensitivity, especially at the onset of an infection. Time consuming laboratory tests can delay adequate treatment for hours. In consequence, empiric antibiotic treatment is often started due to clinically suspected sepsis before the laboratory results are known. A fast and nearly instant diagnostic tool could reduce unnecessary 
exposure to antibiotics in suspected, but unconfirmed sepsis. Blood cultures often yield results 24-48 hours or even later ${ }^{2}$ and are only positive in approximately $0.5 \%^{3,4}$. Rapid diagnosis and prompt initiation of therapy significantly improves outcome ${ }^{5-7}$.

Analysis of volatile organic compounds (VOCs) is an innovative approach for non-invasive diagnostics. VOCs are produced in the body during physiological and pathophysiological processes and are emitted via skin, exhaled air, urine, stool and other body secretions. Many diseases are linked to alterations in specific VOC profiles. Neonates are at high risk for inflammatory disease and sepsis. Necrotizing enterocolitis (NEC) is an acute inflammatory disease of the intestine which primarily affects preterm infants and is a leading cause of morbidity and mortality in the neonatal intensive care unit. Diseases like NEC have been shown to be detectable by VOC detectors ${ }^{8-14}$. Electronic nose devices are based on pattern recognition, measurements can be conducted quickly and at the bedside. Yet those devices do not allow qualitative $\mathrm{VOC}$ analysis, therefore identification of substances as potential biomarkers is not possible. With our established method, ion mobility spectrometry is coupled to multi-capillary columns (MCC/IMS) and allows the measurement of VOC profiles and the specification of individual VOCs using a reference data set. It is also possible to assign individual substances to the detected VOCs. MCC/IMS analyses provide results within a few minutes and can be used for instant bedside diagnostics. Previous studies revealed the high potential of VOC analysis and its application for non-invasive diagnostics. We could demonstrate that VOC profiles can be detected in the incubator atmosphere of neonates, and we were able to differentiate between empty and full incubators using the MCC/IMS ${ }^{15}$. Moreover, we found a potential biomarker for amnion infection syndrome (AIS) using VOC analysis via MCC/IMS ${ }^{16}$.

In this study we aimed to proof if we can generate VOC profiles originating from stool and urine and whether these specimens can be used for diagnostics. Since stool and urine are obtained non-invasively as a waste product, they seem to be suitable for easy diagnostics in preterm infants but also in term infants.

The aim was to establish the method of headspace measurements independent from ambient air for measuring and distinguishing fecal and urine samples and to assign peaks originating from diapers using MCC/IMS as novel noninvasive, cost-effective and precise method.

\section{Methods}

\section{Patients}

This study was performed at the Department of Pediatrics, Saarland University Medical Center, Homburg (Germany) after approval by the Ethical committee Saarland (reference $276 / 17$ ). Written and informed consent was obtained upfront from all parents. All acquired data were recorded and processed in a pseudonymized form. Inclusion criteria were birth weight $<2.000 \mathrm{~g}$ or gestational age $<32$ weeks and treatment on the neonatal care units (NICU) at the Saarland University Hospital, Homburg (Germany). 10 preterm infants were included (Table 1). 


\section{Sample collection and processing}

The diapers were collected daily as part of the morning care routine without generating additional stress for the patients. We used diapers (Pampers, Procter \& Gamble Service GmbH, Schwalbach/ Taunus, Germany) of 10 preterm infants and classified the diaper contents regarding stool consistency, color and quantity using a pediatric stool scale ${ }^{17}$ (Table 1). Fresh diaper pieces served as control. Sample processing was performed according to a standard operating procedure. The surface layer of the diaper was cut into four pieces of a size of $1 \mathrm{~cm} \times 1,5 \mathrm{~cm}$. The pieces were immediately stored at $-80^{\circ} \mathrm{C}$. The samples were thawed at room temperature half an hour before the measurement.

\section{Data assignment /Headspace measurement}

To perform headspace measurements of VOCs derived from the fecal und urine samples, we used an MCC/IMS BreathDiscovery (B \& S Analytik GmbH, Dortmund, Germany). It was placed on a metal cart with a laptop computer and connected to a synthetic air supply. The pre-separation was performed using an OV-5 (5\% - diphenyl, 95\% - dimethylpolysiloxane) multi-capillary column (MCC) (Multichrom Ltd., Novosibirsk, Russia). The device and sampling parameters are given in Supplemental table 1 (Table S1). The methods for VOC analysis were published earlier ${ }^{15}$. A laboratory bottle $(100 \mathrm{~mL})$ heated to $37^{\circ} \mathrm{C}$ served as a sample container. A closed system was established: A large laboratory bottle $(1000 \mathrm{~mL}$, gas reservoir, Schott Duran ${ }^{\circledR}$, DURAN Group GmbH, Wertheim /Main, Germany) was connected to the small bottle (100 mL, Schott Duran®, DURAN Group GmbH, Wertheim /Main, Germany) via a perfluoroalkoxyalkane (PFA) tube that was led through the caps. Both bottles were filled with synthetic air as carrier gas. Another tube connected the cap of the small laboratory bottle and the sampling input of MCC/IMS device (Figure 1). Data was acquired using VOCan v3.7 (B \& S Analytik GmbH, Dortmund, Germany).

\section{Statistical Analysis}

We evaluated the data acquired by MCC/IMS using the software VisualNow 3.7 (B \& S Analytik GmbH, Dortmund, Germany). All peaks were characterized by their specific combination of retention time and drift time (corresponding $1 / \mathrm{K}_{0}$-value). The databank layer (BS-MCC/IMS-analyses database) was used for peak referencing and determination of retention times and $1 / \mathrm{K}_{0}$-values. Peak intensity (in volts) was considered as an indirect measure of compound concentration. A specific threshold was calculated for each peak and comparison. Box-and-Whisker plots and a rank sum test using Mann-Whitney-U test and Bonferroni post hoc analysis correction were performed. The $n$-value was set at $n=10$ for diapers filled with stool, $n=20$ for diapers filled with urine and $n=9$ for empty diapers. The a-level was defined to be 0.05 , the $p$-value (one-tailed) was determined to be $<0.05$. Significant peaks $(p<0.05,95 \%$ confidential interval $(\mathrm{Cl})$ ) were used for further evaluation with decision trees (DT).

\section{Results}


We analyzed a total of 39 diapers filled with stool $(n=10)$ or urine $(n=20)$ and empty diapers $(n=9)$, respectively. We identified 158 signals (peaks) from each comparison such as between empty diapers and diapers with urine, between empty diapers and diapers with stool and between stool and urine, respectively (Figure 2 (D), Supplemental table 3 (Table S3)). We found 17 peaks exclusively assigned to empty diapers in each differentiation. After Bonferroni post hoc analysis correction, Peak P98 showed the highest sensitivity, specificity, positive and negative predictive value with $99,9 \%$ each in a significance level with $\mathrm{p}<0,05(>95 \% \mathrm{Cl}$ ) for empty diapers (Figure 3 (a), Table 2, Supplemental table 2 (Table S2 (a), (c)). Comparing empty diapers to diapers with urine, 48 relevant peaks could be assigned to urine, amongst peak P20 reaching the highest sensitivity, specificity, positive and negative predictive value of $99,9 \%$ each for urine (significance level with $\mathrm{p}<0,01$ (> $99 \% \mathrm{Cl}$ after Bonferroni post hoc analysis correction; Figure 3 (b), Table 2, Supplemental table 2 (Table S2 (a)).

Regarding the comparison between empty diapers and diapers with stool 85 peaks were assigned to stool (Supplemental table 2 (Table S2 (b)). From those peaks, peaks P13 and P83 showed the highest sensitivity, specificity and positive and negative predictive value (99,9\% each) at a significance level with $\mathrm{p}<0,05(>95 \% \mathrm{Cl})$ after Bonferroni post hoc analysis correction (Figure 3 (c), Table 2, Supplemental table 2 (Table S2 (b)).

For the distinction between stool and urine in diapers, 55 relevant peaks with a significance level of at least $p<0,05(>95 \% \mathrm{Cl}$ ) were found (Supplemental table 2 (Table S2 (c)). These peaks differ in their signal intensity; 53 of these peaks were found in diapers with stool and 2 peaks in diapers with urine. Considering those peaks, peak P99 showed the best sensitivity, specificity and positive and negative predictive value of $90 \%, 80 \%, 69,2 \%$ and $94,1 \%$, respectively, in a significance level with $p<0,001(>99,9$ $\% \mathrm{Cl}$ ) for stool (Figure $2(\mathrm{a})-(\mathrm{c}))$. Overall, a total of 20 peaks exclusively assigned to diapers with stool were found in every comparison.

The decision tree reached a sensitivity of $70 \%$ / $95 \%$ / 99,9\% and a positive predictive value of $87,5 \%$ / $86,36 \%$ / 99,9 \% regarding stool, urine, and empty diapers, respectively. Referring to the decision tree, a total of three peaks enabled the differentiation of stool, urine and empty diapers regarding their signal intensities. The peak named P103 allows to differentiate between empty diapers (with a signal intensity $\leq 0,008 \mathrm{~V}$ ) and diapers with stool and urine, respectively (with a signal intensity $>0,008 \mathrm{~V}$ ). Regarding signal intensities $>0,102 \mathrm{~V}$, stool is identified via P160. The Peak P1 enables further differentiation regarding a signal intensity $\leq 0,102 \mathrm{~V}$ : a signal intensity $\leq 0,005 \mathrm{~V}$ stands for urine and a signal intensity $>0,005 \mathrm{~V}$ for stool (Figure $3(\mathrm{D})$ ).

\section{Discussion}

Intensive care treatment and diagnostics are often affiliated with invasive procedures. Establishing a noninvasive, painless method by means of VOCs would be best for vulnerable patients like preterm infants. In the present study we showed that fecal and urine samples in a diaper can be distinguished based on their VOC profile using MCC/IMS. Besides establishing a low contact intensive care treatment, 
VOC analysis via MCC/IMS might allow rapid and precise diagnostics in preterm infants. Current studies using MCC/IMS conducted breath analysis, headspace measurement of biosamples and the measurement of incubators atmosphere ${ }^{15,18-22}$. Chemical substances such as 2-hexanone and butanal detected via MCC/IMS were found to be connected to endotoxic shock or sepsis in rats ${ }^{23}$. Besides, it was shown that bacterial strains of Escherichia coli emitted specific VOCs. Even malignant tumors, as lung cancer seem to be detectable by MCC/IMS ${ }^{18}$.

Other methods such as electronic nose device showed the potential of VOC analysis in stool samples of patients suffering from diseases like inflammatory bowel disease (IBD) and NEC ${ }^{24-26}$. De Meij et al. showed that it was possible to distinguish infants with NEC from healthy controls using an electronic nose device ${ }^{25}$. Stool samples of healthy children's stool samples differed significantly from those suffering from $\mathrm{IBD}^{24}$. Regarding breath analysis, patients suffering from diseases like rheumatoid and psoriatic arthritis could be discriminated from healthy control groups using electronic nose devices ${ }^{27}$. Some studies were able to differentiate various bacteria species ${ }^{28,29}$.

Many studies also conducted analysis on urine samples. Pathogens of urinary tract infections could be differed from each other using electronic nose device (ChemPro 100i, Environics Inc., Mikkeli, Finland). Urine is best suited to detect metabolic disorders such as diabetes: using electronic nose device patients suffering from diabetes could be distinguished from healthy controls. The detection of colorectal cancer seems to be possible by VOC analysis out of urine samples ${ }^{30-32}$. Preterm infants are immature in terms of their metabolism which can lead to metabolic imbalances like hyper- and hypoglycemia. Unfortunately, these irregularities result in frequent blood samplings causing distress and pain ${ }^{33,34}$. Using non-invasive diagnostics like MCC/IMS might facilitate quick and painless detection of glucose levels in urine of premature infants.

We established a method to proceed VOC analysis of fecal and urine samples using MCC/IMS. Further studies should be conducted to examine the potential of ion mobility spectrometry for diagnostic purposes. The method seems to be suitable for $\mathrm{VOC}$ analysis of biosamples as it is able to detect substances with extremely low concentrations (pg/L) ${ }^{35-37}$. As electronic nose devices are only able to detect patterns of VOCs-profiles, MCC/IMS enables to precisely identify substances as well. Our measurements can be conducted quickly within a few minutes at the bedside. Moreover, MCC/IMS is suitable for bedside diagnostics as it can be transported on a cart. It is possible to run the mobile MCC/IMS using a small gas bottle containing synthetic air ${ }^{15}$.

Headspace measurements enable VOC analysis in a closed system and independently from ambient air. Other methods such as VOC analysis from exhaled breath are subject to many influences like oral hygiene, food habits, age and gender. Dead space air potentially causes a dilution of the VOC concentration as well as differences in sample conditions leading to measurement bias. In addition, exhaled breath analysis is difficult to accomplish in neonates as it requires the patient's compliance and the use of a mouthpiece which conflicts with the principle of minimal handling. Compared to other 
methods of VOC analysis such as electronic nose devices which are based on pattern recognition, IMS enables a qualitative analysis of VOCs. The main strength is the ability to assign substances to the spectra. Single substances therefore can be identified as originating from disinfectants or as potential bacterial metabolites ${ }^{38-42}$.

In our study, we identified seven signals that could be assigned to substances using a database (B. Braun Melsungen-Database/ BS-MCC/IMS-analyses database) (Supplemental table 4 (Table S4)). Peak P9 represents 2-pentylfuran and was present in both urine and stool and showed exemplary a high sensitivity/ specificity/ positive predictive value/ negative predictive value (95\% / 99,9\% / 99,9\% / 99,9\%) in a significance level with $\mathrm{p}<0,01$ after Bonferroni post-hoc analysis correction for urine (Supplemental table 2 (Table S2)) and a sensitivity/ specificity/ positive and negative predictive value of $99,9 \%$ each in a significance level with $p<0,05$ after Bonferroni post-hoc analysis correction for stool (Supplemental table 2 (Table S2)) in the respective comparisons to empty diapers. An association between 2-pentylfuran (P9) and Aspergillus was described ${ }^{38,43,44}$. Peak P52 was identified as 5-methyl-2,1-methylethylcyclohexanol. Junger at al. showed that benzonitrile (P32) is associated to bacterial strains and might be an Escherichia coli, Enterobacter cloacae, Proteus mirabilis, Pseudomonas aeruginosa, Serratia marcescens, Staphylococcus epidermidis and Streptococcus pneumoniae ${ }^{40}$. Nevertheless, 5-methyl-2,1methylethylcyclohexanol (P32) assigned to stool in differentiation between empty diapers and stool only showed a sensitivity/ specificity/ positive and negative predictive value of 70\% / 99,9\% / 99,9\% / 75\% (in a significance level with $p<0,05$; Supplemental table 2 (Table S2)). Peak P91 represents 2-hexanone and is a metabolite of the bacterium Escherichia coli that is associated with endotoxin shock and septic shock, respectively, in animal models (FINK et al., 2015; MADDULA et al., 2009). 2-hexanone was exclusively found in stool reaching a sensitivity/ specificity/ positive and negative predictive value of $90 \%$ / 88,9\% / 90\% / 88,9\% (significance level with $p<0,01$; Supplemental table 2 (Table S2)) comparing empty diapers and stool and a sensitivity/ specificity/ positive and negative predictive value of $20 \%$ / 99,9\% / 99,9\% / 71,4\% (significance level with $p<0,05$; Supplemental table 2 (Table S2)) comparing stool and urine, respectively. Those peaks should be reevaluated in further studies with a higher sample count.

One limitation of our study is that we conducted headspace measurement of diaper samples. Additional measurements are required to determine the VOC profiles for stool and urine in the infant's environment or the incubator's atmosphere respectively ${ }^{15}$. Patient size was small in the present study and our results have to be validated on a higher number of patients. Nevertheless, the cohort was homogenous and a lot of attention was paid on sample quality with regard to storing conditions and processing. We processed and stored all samples adhering to a standard operating procedure. Dominianni et al. demonstrated that fecal samples should be stored at $-80^{\circ} \mathrm{C}$ to ensure stability ${ }^{45}$. One study conducted by electronic nose device showed a better signal intensity for samples stored at $-80^{\circ} \mathrm{C}{ }^{46}$. Our diapers containing urine and stool had been stored at $-80^{\circ} \mathrm{C}$ and were thawed prior to the measurements, therefore VOC profiles of native samples might differ from those in our study. Artefacts like the overload of signal intensity must be considered as well. One reason for too intense signals might be ammonia detected in urine. The storage time of stool and urine in the incubator upfront processing might cause an unknown bias and 
has to be investigated more in detail. Furthermore, after all the impact of the gut microbiome on VOC profile remains unknown and has to be considered in future studies.

Overall, a variety of environmental conditions and the sampling processing seem to influence VOC analysis using MCC/IMS. Using additional methods like gas chromatography coupled to mass spectrometry (GC/MS) enables the identification of the analytes and should be realized to perform quantification of the analytes down to the $\mathrm{ng} / \mathrm{L}$ - and $\mathrm{pg} / \mathrm{L}$-range in future. However, our study reveals a high potential for VOC analysis using MCC/IMS. It could contribute to reduce neonatal stress on the NICU through the establishment of a noninvasive diagnostic tool. IMS might become part in the setup of a future low contact incubator in future. We aim to determine volatile biomarkers and/or patterns of volatile biomarkers to use MCC/IMS as a novel, non-invasive and instant diagnostic tool. Our distant objective is to gradually replace conventional, invasive diagnostics by noninvasive methods.

\section{List Of Abbreviations}

$\begin{array}{ll}\text { AIS } & \text { Amnion infection syndrome } \\ \text { BPD } & \text { Bronchopulmonary dysplasia } \\ \text { C } & \text { Celcius } \\ \text { Cl } & \text { Confidential interval } \\ \text { CrP } & \text { C-reactive protein } \\ \text { DT } & \text { Decision tree }\end{array}$

IL-6/ -8 Interleukin- 6/ -8

IMS Ion mobility spectrometry

MCC Multi-capillary column

MCC/IMS Ion mobility spectrometer coupled to multi-capillary column

NEC Necrotizing enterocolitis

NICU...........Neonatal intensive care unit

P Peak

PFA Perfluoroalkoxyalkane

RIP Reactant ion peak

VOCs Volatile organic compounds 


\section{Declarations}

\section{ACKNOWLEDGEMENTS}

We thank Ellen Maurer for her excellent technical help.

\section{AUTHOR CONTRIBUTIONS}

MB: conception and design, sample collection and processing, measurement performing, collection and assembly of data, data analysis and interpretation, manuscript writing, final approval of manuscript.

ET: coordination of sample collection, attainment of parents' written consent, manuscript writing, final approval of manuscript.

NNT: attainment of parents' written consent, manuscript writing, final approval of manuscript.

EK, RS, SM: manuscript writing, final approval of manuscript.

JIB: data analysis and interpretation, manuscript writing, final approval of manuscript.

MZ: conception and design, data analysis and interpretation, financial support, manuscript writing, final approval of manuscript.

SGF: conception and design, data analysis and interpretation, manuscript writing, final approval of manuscript.

All authors have read and agreed to the published version of the manuscript.

\section{DATA AVAILABILITY}

The datasets supporting the conclusions of this article are included within the article or are available from the authors upon reasonable request.

\section{ETHICS STATEMENT}

This study was approved by the Ethics committee at the Saarland Medical Association AZ: $276 / 17$. After written informed consent, the diapers were collected as part of the morning care routine. No additional interaction was performed. All procedures were performed in accordance with relevant guidelines and regulations.

\section{FUNDING}

Funded by a grant from the HOMFOR Foundation of Saarland University Medical School, by the ElseKröner-Fresenius Stiftung, by the Staatskanzlei Saarbrücken, by Centre of Digital Neurotechnologies Saar and by the BMBF (PRIMAL Clinical Study FKZ: 01GL1746D). 


\section{COMPETING INTERESTS}

MB was a student assistant at B. Braun Melsungen AG in cooperation with the Department of General Pediatrics and Neonatology of Saarland University Medical School Homburg from November 2019 to April 2020. The remaining authors declare that they have no competing interests.

\section{AUTHORS' INFORMATION}

\section{Michelle Bous}

Department of Pediatrics and Neonatology

University Children's Hospital of Saarland, Homburg, Germany

Kirrberger Str. 100, D-66421 Homburg

Erol Tutdibi

Department of Pediatrics and Neonatology

University Children's Hospital of Saarland, Homburg, Germany

Kirrberger Str. 100, D-66421 Homburg

Nasenien Nourkami-Tutdibi

Department of Pediatrics and Neonatology

University Children's Hospital of Saarland, Homburg, Germany

Kirrberger Str. 100, D-66421 Homburg

Elisabeth Kaiser

Department of Pediatrics and Neonatology

University Children's Hospital of Saarland, Homburg, Germany

Kirrberger Str. 100, D-66421 Homburg

\section{Regine Stutz}

Department of Pediatrics and Neonatology

University Children's Hospital of Saarland, Homburg, Germany

Kirrberger Str. 100, D-66421 Homburg 
Sascha Meyer

Department of Pediatrics and Neonatology

University Children's Hospital of Saarland, Homburg, Germany

Kirrberger Str. 100, D-66421 Homburg

Jörglngo Baumbach

Department Bio- and Chemical Engineering

Technical University Dortmund, Germany

Emil-Figge-Str. 70, D- 44227 Dortmund

Michael Zemlin

Department of Pediatrics and Neonatology

University Children's Hospital of Saarland, Homburg, Germany

Kirrberger Str. 100, D-66421 Homburg

Sybelle Goedicke-Fritz

Department of Pediatrics and Neonatology

University Children's Hospital of Saarland, Homburg, Germany

Kirrberger Str. 100, D-66421 Homburg

Phone: +496841 1628301

Fax: +49 68411628310

E-Mail: sybelle.goedicke-fritz@uks.eu

\section{References}

1. Howson, C. P., Kinney, M. V., McDougall, L., Lawn, J. E. \& Group, B. T. S. P. B. A. Born too soon: preterm birth matters. Reprod Health 10 Suppl 1, S1, doi:10.1186/1742-4755-10-S1-S1 (2013).

2. Brunkhorst, F. M. \& Schmitz, R. P. Leitliniengerechte Blutkulturdiagnostik bei Sepsis. intensiv 21, 261263 (2013).

3. Mikhael, M., Brown, L. S. \& Rosenfeld, C. R. Serial neutrophil values facilitate predicting the absence of neonatal early-onset sepsis. J Pediatr 164, 522-528.e521-523, doi:10.1016/j.jpeds.2013.10.080 
(2014).

4. Escobar, G. J. et al. Stratification of risk of early-onset sepsis in newborns $\geq 34$ weeks' gestation. Pediatrics 133, 30-36, doi:10.1542/peds.2013-1689 (2014).

5. Flidel-Rimon, O., Leibovitz, E., Eventov Friedman, S., Juster-Reicher, A. \& Shinwell, E. S. Is lumbar puncture (LP) required in every workup for suspected late-onset sepsis in neonates? Acta Paediatr 100, 303-304, doi:10.1111/j.1651-2227.2010.02012.x (2011).

6. Raju, T. N., Higgins, R. D., Stark, A. R. \& Leveno, K. J. Optimizing care and outcome for late-preterm (near-term) infants: a summary of the workshop sponsored by the National Institute of Child Health and Human Development. Pediatrics 118, 1207-1214, doi:10.1542/peds.2006-0018 (2006).

7. Cohen-Wolkowiez, M. et al. Early and late onset sepsis in late preterm infants. Pediatr Infect Dis J 28, 1052-1056, doi:10.1097/inf.0b013e3181acf6bd (2009).

8. Handrick, W. \& Huppertz, H.-I. in Duale Reihe Pädiatrie (eds Ludwig Gortner \& Sascha Meyer) (Georg Thieme Verlag, 2018).

9. Zeuzem, S., Schöffauer, M. \& Rösch, W. in Duale Reihe Innere Medizin (eds Keikawus Arastéh et al.) (Georg Thieme Verlag, 2018).

10. Bin-Nun, A. et al. Oral probiotics prevent necrotizing enterocolitis in very low birth weight neonates. J Pediatr 147, 192-196, doi:10.1016/j.jpeds.2005.03.054 (2005).

11. Deshpande, G., Rao, S. \& Patole, S. Probiotics for prevention of necrotising enterocolitis in preterm neonates with very low birthweight: a systematic review of randomised controlled trials. The Lancet 369, 1614-1620, doi:https://doi.org/10.1016/S0140-6736(07)60748-X (2007).

12. Arrieta, M. C. et al. Early infancy microbial and metabolic alterations affect risk of childhood asthma. Sci Transl Med 7, 307ra152, doi:10.1126/scitransImed.aab2271 (2015).

13. Fujimura, Kei E. \& Lynch, Susan V. Microbiota in Allergy and Asthma and the Emerging Relationship with the Gut Microbiome. Cell Host \& Microbe 17, 592-602, doi:https://doi.org/10.1016/j.chom.2015.04.007 (2015).

14. Johnson, C. C. et al. Antibiotic exposure in early infancy and risk for childhood atopy. Journal of Allergy and Clinical Immunology 115, 1218-1224, doi:https://doi.org/10.1016/j.jaci.2005.04.020 (2005).

15. Steinbach, J. et al. Bedside Measurement of Volatile Organic Compounds in the Atmosphere of Neonatal Incubators Using Ion Mobility Spectrometry. Front Pediatr 7, 248-248, doi:10.3389/fped.2019.00248 (2019).

16. Goedicke-Fritz, S. et al. Detection of Volatile Organic Compounds as Potential Novel Biomarkers for Chorioamnionitis - Proof of Experimental Models. Front Pediatr 9, 698489-698489, doi:10.3389/fped.2021.698489 (2021).

17. Koletzko, S., Otte, S. \& Klucker, E. Stuhltests in der pädiatrischen Gastroenterologie. Monatsschr Kinderheilkd, 572-580 (2017). 
18. Darwiche, K., Baumbach, J. I., Sommerwerck, U., Teschler, H. \& Freitag, L. Bronchoscopically obtained volatile biomarkers in lung cancer. Lung 189, 445-452, doi:10.1007/s00408-011-9324-1 (2011).

19. Hüppe, T. et al. Volatile Organic Compounds in Patients With Acute Kidney Injury and Changes During Dialysis*. Critical Care Medicine 47 (2019).

20. Buchinger, H. et al. Minimal retarded Propofol signals in human breath using ion mobility spectrometry. International Journal for Ion Mobility Spectrometry 16, 185-190, doi:10.1007/s12127012-0118-9 (2013).

21. Kreuder, A. E. et al. Characterization of propofol in human breath of patients undergoing anesthesia. International Journal for Ion Mobility Spectrometry 14, 167-175, doi:10.1007/s12127-011-0080-y (2011).

22. Perl, T. et al. Determination of serum propofol concentrations by breath analysis using ion mobility spectrometry. Br J Anaesth 103, 822-827, doi:10.1093/bja/aep312 (2009).

23. Fink, T. et al. Volatile organic compounds during inflammation and sepsis in rats: a potential breath test using ion-mobility spectrometry. Anesthesiology 122, 117-126, doi:10.1097/ALN.0000000000000420 (2015).

24. de Meij, T. G. et al. Faecal gas analysis by electronic nose as novel, non-invasive method for assessment of active and quiescent paediatric inflammatory bowel disease: Proof of principle study. J Crohns Colitis, doi:10.1016/j.crohns.2014.09.004 (2014).

25. de Meij, T. G. et al. Early Detection of Necrotizing Enterocolitis by Fecal Volatile Organic Compounds Analysis. J Pediatr 167, 562-567.e561, doi:10.1016/j.jpeds.2015.05.044 (2015).

26. Buijck, M. et al. Sniffing Out Paediatric Gastrointestinal Diseases: The Potential of Volatile Organic Compounds as Biomarkers for Disease. Journal of pediatric gastroenterology and nutrition 63, 585591, doi:10.1097/MPG.0000000000001250 (2016).

27. Brekelmans, M. P. et al. Smelling the Diagnosis: The Electronic Nose as Diagnostic Tool in Inflammatory Arthritis. A Case-Reference Study. PLoS One 11, e0151715, doi:10.1371/journal.pone.0151715 (2016).

28. Thaler, E. R. et al. Use of an electronic nose for detection of biofilms. American journal of rhinology 22, 29-33, doi:10.2500/ajr.2008.22.3126 (2008).

29. Saviauk, T. et al. Electronic Nose in the Detection of Wound Infection Bacteria from Bacterial Cultures: A Proof-of-Principle Study. Eur Surg Res 59, 1-11, doi:10.1159/000485461 (2018).

30. Arasaradnam, R. P. et al. Detection of colorectal cancer (CRC) by urinary volatile organic compound analysis. PLoS One 9, e108750, doi:10.1371/journal.pone.0108750 (2014).

31. Ma, Y. L. et al. Ultra-high performance liquid chromatography-mass spectrometry for the metabolomic analysis of urine in colorectal cancer. Dig Dis Sci 54, 2655-2662, doi:10.1007/s10620008-0665-4 (2009).

32. McFarlane, M. et al. Urinary volatile organic compounds and faecal microbiome profiles in colorectal cancer. Colorectal Dis 21, 1259-1269, doi:10.1111/codi.14739 (2019). 
33. Adamkin, D. H. Postnatal glucose homeostasis in late-preterm and term infants. Pediatrics $127,575-$ 579, doi:10.1542/peds.2010-3851 (2011).

34. Mitanchez, D. Glucose regulation in preterm newborn infants. Horm Res $68,265-271$, doi:10.1159/000104174 (2007).

35. Baumbach, J. I. Process analysis using ion mobility spectrometry. Analytical and Bioanalytical Chemistry 384, 1059-1070, doi:10.1007/s00216-005-3397-8 (2006).

36. Cumeras, R., Figueras, E., Davis, C. E., Baumbach, J. I. \& Gràcia, I. Review on ion mobility spectrometry. Part 1: current instrumentation. The Analyst 140, 1376-1390, doi:10.1039/c4an01100g (2015).

37. Ruzsanyi, V. et al. Detection of human metabolites using multi-capillary columns coupled to ion mobility spectrometers. J Chromatogr A 1084, 145-151 (2005).

38. Bos, L. D., Sterk, P. J. \& Schultz, M. J. Volatile metabolites of pathogens: a systematic review. PLoS Pathog 9, e1003311, doi:10.1371/journal.ppat.1003311 (2013).

39. Chambers, S. T., Bhandari, S., Scott-Thomas, A. \& Syhre, M. Novel diagnostics: progress toward a breath test for invasive Aspergillus fumigatus. Medical mycology 49, S54-S61 (2011).

40. Junger, M. et al. Ion mobility spectrometry for microbial volatile organic compounds: a new identification tool for human pathogenic bacteria. Applied microbiology and biotechnology 93, 2603-2614, doi:10.1007/s00253-012-3924-4 (2012).

41. Maddula, S., Blank, L. M., Schmid, A. \& Baumbach, J. I. Detection of volatile metabolites of Escherichia coli by multi capillary column coupled ion mobility spectrometry. Analytical and bioanalytical chemistry 394, 791-800, doi:10.1007/s00216-009-2758-0 (2009).

42. Syhre, M., Scotter, J. M. \& Chambers, S. T. Investigation into the production of 2-Pentylfuran by Aspergillus fumigatus and other respiratory pathogens in vitro and human breath samples. Medical Mycology 46, 209-215 (2008).

43. Chambers, S. T., Bhandari, S., Scott-Thomas, A. \& Syhre, M. Novel diagnostics: progress toward a breath test for invasive Aspergillus fumigatus. Med Mycol 49 Suppl 1, S54-61, doi:10.3109/13693786.2010.508187 (2011).

44. Syhre, M., Scotter, J. M. \& Chambers, S. T. Investigation into the production of 2-Pentylfuran by Aspergillus fumigatus and other respiratory pathogens in vitro and human breath samples. Med Mycol 46, 209-215, doi:10.1080/13693780701753800 (2008).

45. Dominianni, C., Wu, J., Hayes, R. B. \& Ahn, J. Comparison of methods for fecal microbiome biospecimen collection. BMC Microbiol 14, 103, doi:10.1186/1471-2180-14-103 (2014).

46. Esfahani, S. et al. Variation in Gas and Volatile Compound Emissions from Human Urine as It Ages, Measured by an Electronic Nose. Biosensors 6, doi:10.3390/bios6010004 (2016).

\section{Tables}


Table 1: Sample classification. We used diapers (Pampers, Procter \& Gamble Service GmbH,

Schwalbach/ Taunus, Germany) from a total of 10 preterm infants. For each sample, infants` ID, sex, gestational age, birth weight and diaper size are indicated ( $f$ - female, $m$ - male, $d$ - day). (a) Parameters of the samples containing urine. (b) Samples with stool are classified additionally regarding a stool chart ${ }^{17}$ referring to the amount, consistency and color of the stool samples (Amount - 1: smear, 2: up to $25 \%$, 3: 25-50 \%, 4: more than 50 \%; Consistency - A: watery, B: soft, C: formed, D: hard; Color - I: yellow, II: orange, III: green, IV: brown, V: meconium, VI: white).

\begin{tabular}{|c|c|c|c|c|c|}
\hline Infant ID & Sex & Gestational age [weeks] & Birth weight [g] & Sampling day & Diaper size \\
\hline 22 & $f$ & $28+4$ & 980 & D7 & 0 \\
\hline 22 & $f$ & $28+4$ & 980 & D8 & 0 \\
\hline 22 & $f$ & $28+4$ & 980 & D9 & 1 \\
\hline 26 & $\mathrm{~m}$ & $29+6$ & 950 & D9 & 0 \\
\hline 26 & $\mathrm{~m}$ & $29+6$ & 950 & D10 & 0 \\
\hline 28 & $\mathrm{~m}$ & $32+2$ & 1360 & D6 & 0 \\
\hline 28 & $\mathrm{~m}$ & $32+2$ & 1360 & D8 & 0 \\
\hline 28 & $\mathrm{~m}$ & $32+2$ & 1360 & D10 & 1 \\
\hline 29 & $f$ & $32+2$ & 1605 & D4 & 0 \\
\hline 29 & $f$ & $32+2$ & 1605 & D6 & 0 \\
\hline 29 & $f$ & $32+2$ & 1605 & D7 & 0 \\
\hline 29 & $f$ & $32+2$ & 1605 & D8 & 0 \\
\hline 30 & $\mathrm{~m}$ & $32+2$ & 1570 & D3 & 0 \\
\hline 30 & $\mathrm{~m}$ & $32+2$ & 1570 & D5 & 1 \\
\hline 31 & $f$ & $32+2$ & 1405 & D7 & 0 \\
\hline 32 & $\mathrm{~m}$ & $33+2$ & 1580 & D1 & 0 \\
\hline 32 & $\mathrm{~m}$ & $33+2$ & 1580 & D2 & 0 \\
\hline 32 & $\mathrm{~m}$ & $33+2$ & 1580 & D4 & 0 \\
\hline 32 & $m$ & $33+2$ & 1580 & D7 & 0 \\
\hline 32 & $\mathrm{~m}$ & $33+2$ & 1580 & D8 & 0 \\
\hline
\end{tabular}


b:

Stool

$\begin{array}{lllllll}\text { Infant } & \text { Sex } & \begin{array}{l}\text { Gestational } \\ \text { age [weeks] }\end{array} & \begin{array}{l}\text { Birth } \\ \text { weight } \\ \text { [g] }\end{array} & \begin{array}{l}\text { Sampling } \\ \text { day }\end{array} & \begin{array}{l}\text { Diaper } \\ \text { size }\end{array} & \text { Amount Consistency Color }\end{array}$

\begin{tabular}{lllllllll}
21 & M & $28+4$ & 990 & D7 & 0 & 4 & B & II \\
21 & M & $28+4$ & 990 & D8 & 0 & 4 & B & II \\
\hline 21 & M & $28+4$ & 990 & D10 & 0 & 4 & B & II \\
\hline 22 & F & $28+4$ & 980 & D1 & 0 & 3 & C & II \\
\hline 22 & F & $28+4$ & 980 & D2 & 0 & 4 & C & II/IV \\
\hline 24 & F & $28+6$ & 1310 & D3 & 0 & 3 & A+C & III/IV \\
\hline 24 & F & $28+6$ & 1310 & D9 & 0 & 4 & B & II \\
\hline 25 & F & $30+5$ & 1610 & D4 & 0 & 1 & A & I \\
\hline 25 & F & $30+5$ & 1610 & D9 & 0 & 4 & B & II \\
\hline 32 & M & $33+2$ & 1580 & D3 & 0 & 4 & B & II
\end{tabular}

Table 2: Statistical analyses for the model set and validation set for peaks P98, P20 and P83.

The peaks P98, P20 and P83 are characterized regarding 
$\begin{array}{lll}\text { P98 } & \text { P20 } & \text { P83 }\end{array}$

\begin{tabular}{|c|c|c|c|c|c|c|}
\hline Best direction & $\begin{array}{l}\text { Empty } \\
\text { diaper > } \\
\text { Stool }\end{array}$ & $\begin{array}{l}\text { Empty } \\
\text { diaper > } \\
\text { Urine }\end{array}$ & $\begin{array}{l}\text { Urine > } \\
\text { Empty } \\
\text { diaper }\end{array}$ & $\begin{array}{l}\text { Urine > } \\
\text { Stool }\end{array}$ & $\begin{array}{l}\text { Stool > } \\
\text { Empty } \\
\text { diaper }\end{array}$ & $\begin{array}{l}\text { Stool > } \\
\text { Urine }\end{array}$ \\
\hline Best threshold & 0,02 & 0,02 & 0,018 & 0,018 & 0,038 & 0,079 \\
\hline Classified right & 19 & 29 & 29 & 21 & 19 & 26 \\
\hline Classified wrong & 0 & 0 & 0 & 9 & 0 & 4 \\
\hline True positive & 10 & 20 & 20 & 1 & 10 & 6 \\
\hline False positive & 0 & 0 & 0 & 0 & 0 & 0 \\
\hline True negative & 9 & 9 & 9 & 20 & 9 & 20 \\
\hline False negative & 0 & 0 & 0 & 9 & 0 & 4 \\
\hline Sensitivity [\%] & 99,9 & 99,9 & 99,9 & 10 & 99,9 & 60 \\
\hline Specificity [\%] & 99,9 & 99,9 & 99,9 & 99,9 & 99,9 & 99,9 \\
\hline $\begin{array}{l}\text { Positive } \\
\text { predictive value } \\
\text { [\%] }\end{array}$ & 99,9 & 99,9 & 99,9 & 99,9 & 99,9 & 99,9 \\
\hline $\begin{array}{l}\text { Negative } \\
\text { predictive value } \\
\text { [\%] }\end{array}$ & 99,9 & 99,9 & 99,9 & 69 & 99,9 & 83,3 \\
\hline Accuracy [\%] & 99,9 & 99,9 & 99,9 & 70 & 99,9 & 86,7 \\
\hline $\begin{array}{l}\text { Significance level } \\
\text { (Mann Whitney } \\
\text { U) }\end{array}$ & $<0.001$ & $<0.001$ & $<0.001$ & - & $<0.001$ & $<0.05$ \\
\hline $\begin{array}{l}\text { Significance } \\
\text { level }\end{array}$ & $<0.05$ & $<0.01$ & $<0.01$ & - & $<0.05$ & - \\
\hline $\begin{array}{l}\text { (Bonferroni } \\
\text { correction) }\end{array}$ & & & & & & \\
\hline
\end{tabular}

\section{Figures}




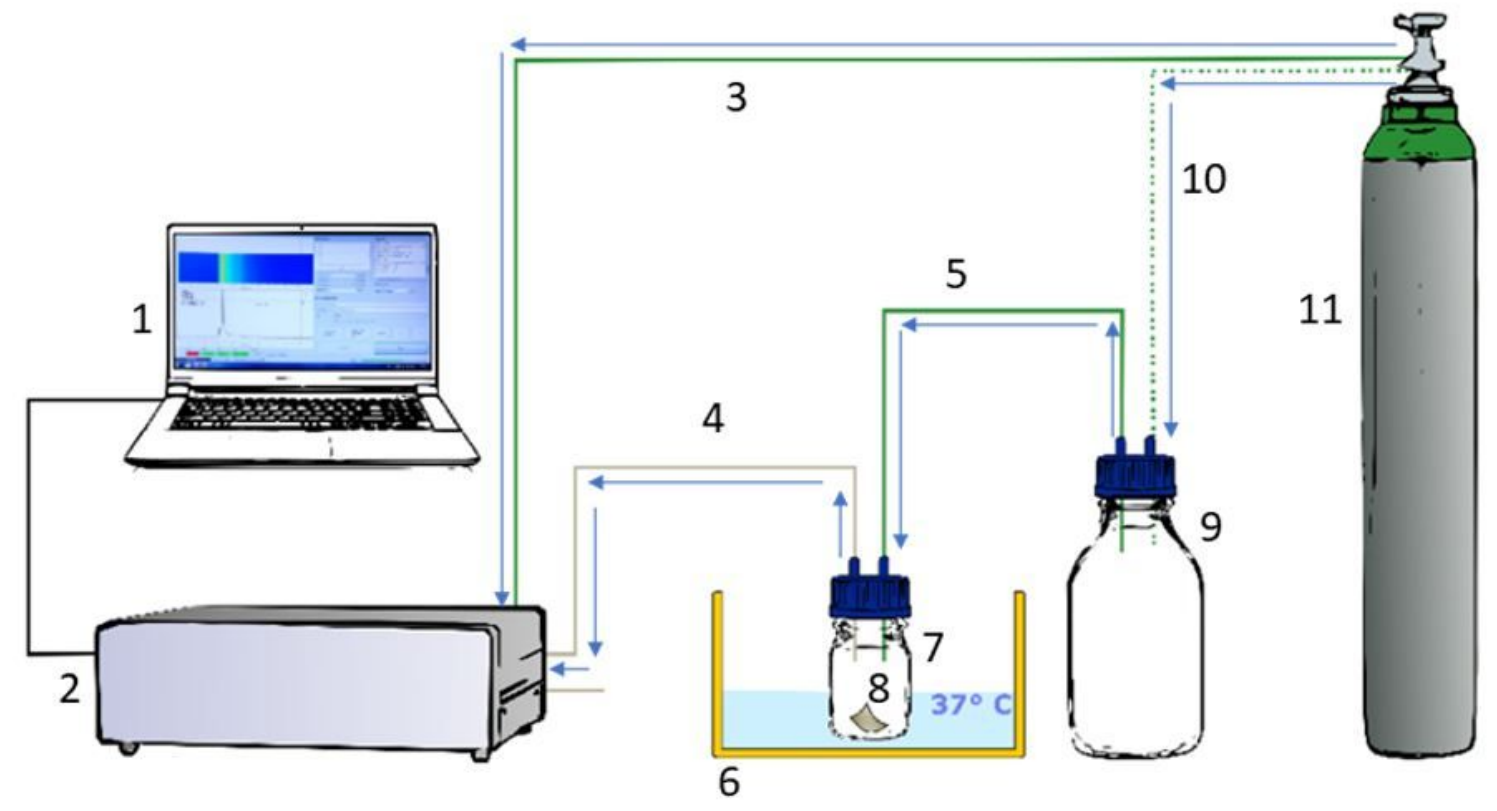

\section{Figure 1}

\section{Experimental design.}

We used an MCC/IMS BreathDiscovery (B \& S Analytik GmbH, Dortmund, Germany) to perform headspace measurements of VOCs derived from the fecal und urine samples. (1) Laptop; (2) MCC/IMS BreathDiscovery (B \& S Analytik GmbH, Dortmund, Germany); (3) Connecting tube between MCC/IMS and synthetic air supply gas bottle; (4) Connecting tube between MCC/IMS and sample container; (5) Connecting tube between gas reservoir and sample container; (6) Water bath; (7) Sample container: small laboratory bottle (100 mL); (8) Diaper sample; (9) Gas reservoir: large laboratory bottle (1000 mL); (10) Connecting tube between synthetic air supply gas bottle and gas reservoir; (11) Synthetic air supply gas bottle; PFA tubes (green) as drift gas supply; PFA tube (grey) as sample input. Data acquisition was directed and recorded using VOCan v3.7 (B \& S Analytik GmbH, Dortmund, Germany). 


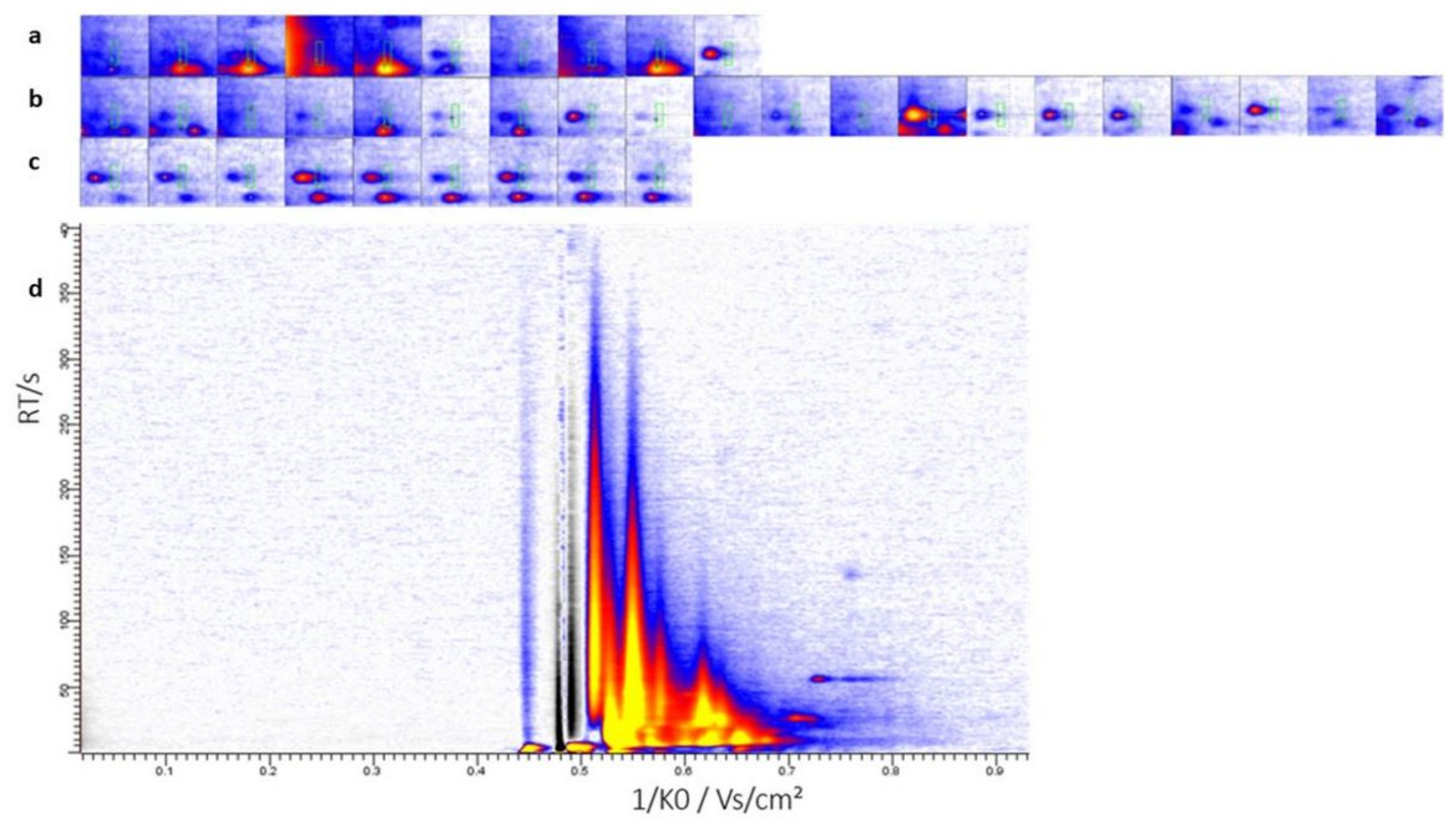

Figure 2

Intensity distribution of peak P99 and representative chromatogram.

(a)-(c) Shown are the cutouts of MCC/IMS-chromatograms of peak P99 for every single measurement. In (a) we showed diapers with stool, in (b) diapers with urine and in (c) empty diapers as a control.

(d) Heatmap: The $y$-axis of the heat map represents the retention time RT and $x$-axis represents the inverse ion mobility $1 / K 0$, a transformation of the drift time. The colors display the signal intensities with increasing values from white over blue and red to yellow. White $=$ no signal, blue $=$ low signal, red $=$ medium signal, yellow $=$ high signal. 

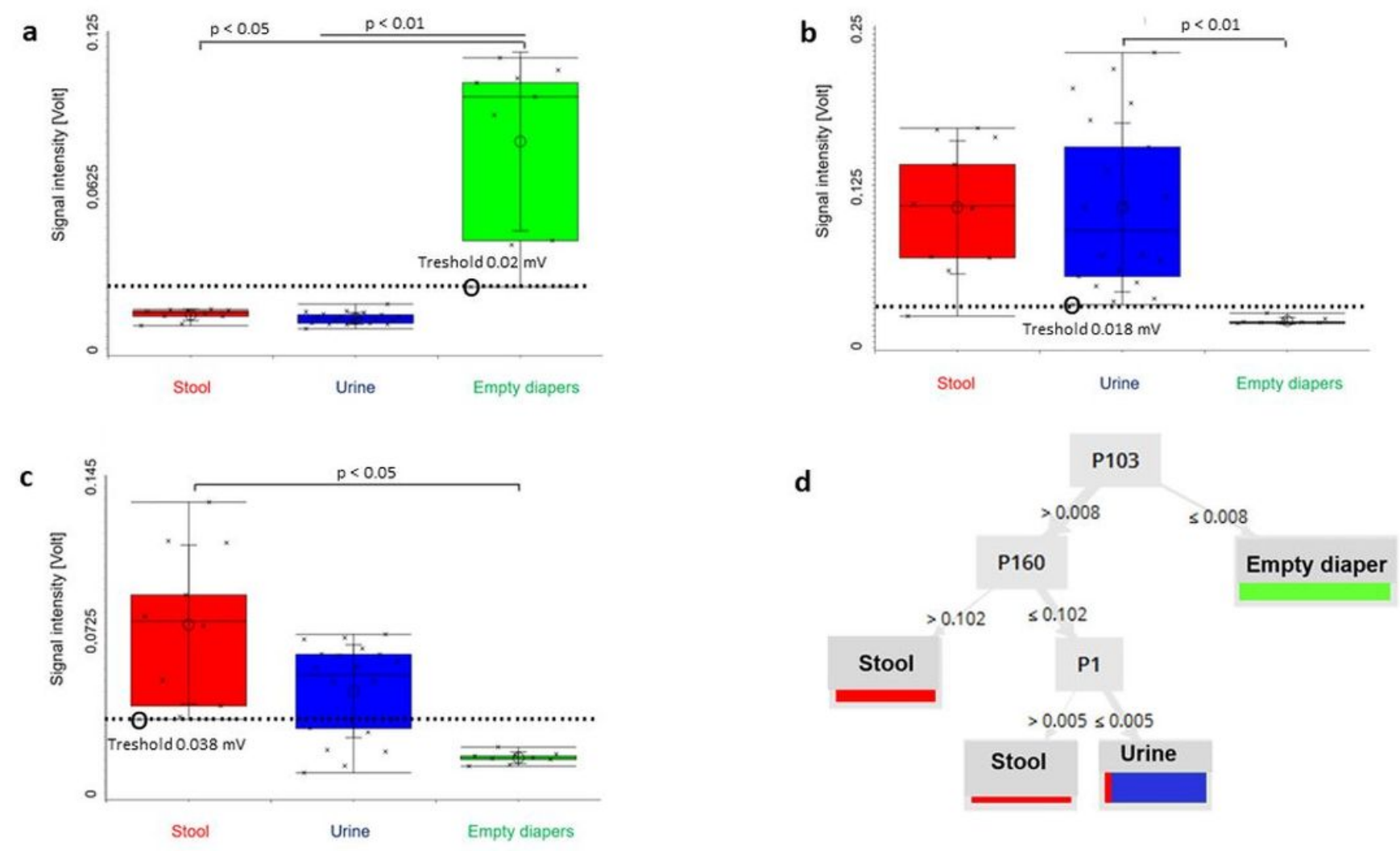

Figure 3

\section{Box-and-Whisker Plot of relevant peaks of the comparison of empty diapers and diapers with urine or stool, respectively.}

Measurements are marked with crosses. (a) Peak P98 exclusively assigned to empty diapers showed the highest sensitivity, specificity, positive and negative predictive value with 99,9\% each (significance level with $\mathrm{p}<0,05(>95 \% \mathrm{Cl})$ ). (b) After Bonferroni post hoc analysis correction Peak P20 reached the best sensitivity, specificity, positive and negative predictive value (99,9\% each, $p<0,01(>99 \% \mathrm{Cl})$ ) regarding urine in the differentiation between empty diapers and diapers with urine. (c) Comparing empty diapers and diapers with stool, peak P83 was assigned to a sensitivity, specificity, positive and negative predictive value of $99,9 \%$ each in a significance level with $p<0,05(>95 \% \mathrm{Cl})$ after Bonferroni post hoc analysis correction. (d) A decision tree based on three compounds is shown. Samples are grouped according to the means of the peak intensity of each compound, at which point, the maximum number of samples are classified correctly. Relative numbers of classified all samples stool are blue, urine are green and empty diapers are red. Discrimination of the three groups is possible.

The decision tree reached a sensitivity of $70 \%$ / $95 \%$ / 99,9\% and a positive predictive value of $87,5 \%$ / $86,36 \%$ / 99,9\% regarding stool, urine, and empty diapers, respectively. 


\section{Supplementary Files}

This is a list of supplementary files associated with this preprint. Click to download.

- Supplement140122.pdf 